\title{
Asymptotic Behavior of $h p$-HGS ( $h p$-Adaptive Finite Element Method Coupled with the Hierarchic Genetic Strategy) by Solving Inverse Problems
}

\author{
Robert Schaefer ${ }^{1}$ and Barbara Barabasz ${ }^{2}$ \\ ${ }^{1}$ Department of Computer Science \\ 2 Department of Modeling and Information Technology, \\ AGH University of Science and Technology, \\ Al. Mickiewicza 30, 30-059 Cracow, Poland, \\ schaefer@agh.edu.pl \\ barabasz@metal.agh.edu.pl
}

\begin{abstract}
The new $h p$-HGS multi-deme, genetic strategy for economic solving of the parametric inverse problems is introduced. The inverse problems under consideration are formulated as the global optimization ones, where the objective express the discrepancy between the computed and measured energy. The efficiency of the proposed strategy results from the coupled adaptation of the accuracy of solving optimization problem and the accuracy of $h p$-FEM direct problem solver. The asymptotic analysis allows to estimate the expected computational cost of $h p$-HGS and to show its advantage over the single population SGA algorithm as well as over the HGS strategy without the FEM error scaling.
\end{abstract}

\section{Introduction}

The class of direct problems under considerations is defined by the abstract variational equation describing the sample physical phenomena (e.g. the variational equations of the linear elasticity described in [3]):

$$
\left\{\begin{array}{l}
u \in u_{0}+V \\
b(d ; u, v)=l(v) \forall v \in V
\end{array}\right.
$$

where $u_{0}$ is the shift of the Dirichlet boundary conditions and $V$ is the proper Sobolev space. The form of functionals $b, l$ depend of the physical phenomena and of its parameter $d \in \mathcal{D}$, where $\mathcal{D}$ is the regular compact in $\mathbb{R}^{N}, N<+\infty$. For symmetric and positively defined $b$, the variational problem (1) can be formulated as the minimization one

$$
\left\{\begin{array}{l}
u \in u_{0}+V \\
\min \{E(d ; u)\},
\end{array}\right.
$$

where the functional $E(d ; u)=\frac{1}{2} b(d ; u, u)-l(u)$ stands for the total energy of the modeled system. 
One of the most effective numerical method of solving the above problem is the $h p$-adaptive Finite Element Method ( $h p$-FEM) which approximates its solution by the sequence of finite dimensional problems (see Demkowicz [4). The coarse mesh solution $u_{h, p} \in V_{h, p}$ that satisfies

$$
\left\{\begin{array}{l}
u_{h, p} \in u_{0}+V_{h, p} \\
b\left(d ; u_{h, p}, v_{h, p}\right)=l\left(v_{h, p}\right) \forall v_{h, p} \in V_{h, p}
\end{array}\right.
$$

is computed in each step of this strategy. Moreover the fine mesh solution $u_{\frac{h}{2}, p+1} \in V_{\frac{h}{2}, p+1}$ that satisfies the equation similar to (3) in the space $V_{\frac{h}{2}, p+1}$ is computed. Notice that both approximate solution spaces satisfy $V_{h, p} \subset V_{\frac{h}{2}, p+1} \subset$ $V$. Formally, the solutions to (11), (2), (3) depend also on the parameter $d \in \mathcal{D}$.

Based on the relative $h p$-FEM error analysis (see Demkowicz [4])

$$
\operatorname{err}_{F E M}(d)=\left\|u_{h, p}(d)-u_{\frac{h}{2}, p+1}(d)\right\|_{E}
$$

the final solution of the $h p-\mathrm{FEM}$ step is established. It persists the modification introduced by $u_{\frac{h}{2}, p+1}$ only in elements, where this error is large. The norm $\|\cdot\|_{E}$ defined on the space $V$ expresses the energy of its argument (see e.g. Ciarlet [3]).

We assume that all above problems (11), (2), (3) are well posed, i.e. they posses the unique solution for all possible values of the admissible parameter $d \in \mathcal{D}$.

The inverse problem under consideration leads to encountering the unknown parameter $\hat{d} \in \mathcal{D}$ while the energy $J(\hat{d})=E(\hat{d} ; u)$ of the exact solution $u \in V$ to (11) is known (e.g. it is measured during the laboratory test). It can be formulated as follows:

Find $\hat{g} \in \mathcal{D}$ such that :

$$
\lim _{h \rightarrow 0, p \rightarrow+\infty}\left|J_{h, p}(\hat{g})-J(\hat{d})\right| \leq \lim _{h \rightarrow 0, p \rightarrow+\infty}\left|J_{h, p}(g)-J(\hat{d})\right|
$$

The above problem is the global optimization one with the admissible set of solutions $\mathcal{D}$. The quantities $\hat{g}, g$ represent approximated parameters and $\hat{d}$ the exact parameter of the inverse problem which we are looking for. Moreover $J_{h, p}(g)=E\left(g ; u_{h, p}(g)\right)$ is the energy of solution $u_{h, p}(g)$ obtained by $h p$-FEM. We assume moreover, that the sufficient conditions are satisfied (see Demkowicz [4]) that the expression $\left|J_{h, p}(g)-J(\hat{d})\right|$ has a finite limit for all $g, \hat{d} \in \mathcal{D}$, while $h \rightarrow 0, p \rightarrow+\infty$.

One of the main difficulties of the above optimization problem is caused by presence of more than one global extreme or local extremes with the objective very close to the minimal one. Moreover, the cost of the objective evaluation $\left|J_{h, p}(g)-J(\hat{d})\right|$ is large, because of the large cost of iterative solving of direct problem. Please, notice that this cost strongly depends on the required accuracy of the direct problem solving. The global search with the exceptionally low computational cost (counting in the number of objective evaluations) is then desirable for solving the inverse problem (5). 
We propose the new strategy $h p$-HGS which is based on the economic Hierarchic Genetic Strategy (HGS) (see Schaefer, Kołodziej [10]) with the adaptive solution accuracy. It offers relatively small number of fitness function calls and ability of global search of solution, especially in case of many local extremes. The proposed strategy additionally decreases the total computational cost by scaling the accuracy of the direct problem solving with respect to varying accuracy of the inverse problem solution.

The HGS proceeds tree-structured, dynamically changing set of dependent demes. The depth of HGS tree is limited by $m<+\infty$. All demes work asynchronously and are synchronized by the message-passing mechanism if necessary. The evolution of each deme is governed by the separate instance of the Simple Genetic Algorithm (SGA) (see Vose [15]).

The low-order demes (closer to the root) perform more chaotic search with the lower accuracy, while the demes of higher order perform the more accurate, local search. The various search accuracy is obtained by the various encoding precision and by the different length binary strings as the genotypes in demes of different order. The unique deme of the first order (root) utilizes the shortest genotypes, while the leafs utilizes the longest ones. To obtain the search coherency for demes of different order the special kind of hierarchical, nested encoding is used. First the densest mesh of phenotypes in $\mathcal{D}$ for the demes of $m$-th order is defined. Next the meshes for the lower order demes are recursively defined by selecting some nodes from the previous ones. The maximum diameter of the mesh $\delta_{j}$ associated with the demes of the order $j$ determines the search accuracy at this level of the HGS tree. Of course $\delta_{1}>, \ldots,>\delta_{m}$.

Each deme expecting leaf-demes sprouts the new child-deme after the constant number of genetic epochs $K$ called the metaepoch. The child-deme is activated in the promising region of the evolutionary landscape surrounding the best fitted individual distinguished from the parental deme at the end of the metaepoch.

HGS implements also two mechanisms that allow to reduce the search redundancy. The first one called conditional sprouting disable to sprout new deme in the region already occupied or explored by the brother-deme (another childdeme of the same order sprouted by the same parent). The second mechanism called branch reduction reduces the branches of the same order that perform the search in the common landscape region or in the region already explored.

The HGS details and various implementations of this strategy as well as its high efficiency by solving global optimization problems are presented in [6], [1], [8], 10], [16], [7].

\section{Relation Between the $h p$-FEM Error and HGS Objective Function Error}

Let us apply HGS for solving the inverse problem (5). The fitness function for the particular deme should be based on the energy error

$$
e_{h, p}(g)=\left|J_{h, p}(g)-J(\hat{d})\right|
$$


computed by using $h p-\mathrm{FEM}$ which approximate the objective function of the global optimization problem (5) for the particular values of $h$ and $p$. As previously, $\hat{d} \in \mathcal{D}$ denotes the exact parameter value and $J(\hat{d})$ the known, exact energy of the exact solution while $J_{h, p}(g)$ the approximated value of energy computed by $h p$-FEM with respect to the parameter value $g \in \mathcal{D}$ obtained from the HGS individuals' genotype.

Let us assume for a while that $g$ is constant and represents the parameter value decoded from the genotype that appears in the HGS deme of the $j$-th order, $j \in\{1, \ldots, m\}$. The regression of the error (6) while improving the FEM approximation may be evaluated as follows (see Lemma 2 in [12]):

$$
e_{\frac{h}{2}, p+1}(g) \leq \frac{1}{2}\left\|u_{\frac{h}{2}, p+1}(g)-u_{h, p}(g)\right\|_{E}^{2}+\left|J_{h, p}(g)-J(\hat{d})\right|
$$

where $e_{\frac{h}{2}, p+1}(g)=\left|J_{\frac{h}{2}, p+1}(g)-J(\hat{d})\right|$. Further using the formula (8) in [12] we may obtain

$$
e_{\frac{h}{2}, p+1}(g) \leq\left\|u_{\frac{h}{2}, p+1}(g)-u_{h, p}(g)\right\|_{E}^{2}+\left\|u(g)-u_{h, p}(g)\right\|_{E}^{2}+L|g-\hat{d}|
$$

where $L$ stands for the Lipshitz constants of the functional $J$ and $|g-\hat{d}|$ is the error of the inverse problem solution that characterizes the individuals belonging to the HGS demes of the $j$-th order. It is easy to observe, that $|g-\hat{d}|$ corresponds to $\delta_{j}$. The above formula shows, that the error of the energy evaluation over the fine FEM mesh is restricted by the relative FEM error on the coarse FEM mesh solution with respect to the fine mesh solution plus the absolute FEM error over the coarse FEM mesh plus the accuracy of the proper HGS branch.

\section{$3 h p$-HGS Definition}

The main idea of $h p$-HGS is to adjust dynamically the accuracy of the objective computation to the particular value of the parameter $g$ encoded in the individuals' genotype as well as for the inverse problem error that characterizes the current HGS branch. It may be obtained by balancing the components of the FEM error given by the right hand side of the formula (8), assuming $\delta_{j}$ as the accuracy of inverse problem solving by the branch of $j$-th order. We will perform then the $h p$-adaptation of the FEM solution of the direct problem while the quantity $\frac{\operatorname{err}_{F E M}}{\delta_{j}}$ is greater then the assumed Ratio, which stands for the parameter of this strategy.

Notice, that no matter how the fitness of the individual $i$ is computed by iterative process of the $h p-\mathrm{FEM}$ adaptation, the fitness function $f_{j}$ is well defined for all individuals in branches of $j$-th order (it is not a random variable).

The draft of the single $h p$-HGS deme activity is stressed in the pseudo-code Algorithm 1 The function branch_stop_condition $(P)$ returns true if it detects the lack of evolution progress of the current deme $P$. The separate module continuously checks whether the satisfactory solution was found or $h p$-HGS 
could not find more local extremes. If yes, the global_stop_condition signal is send to all computing demes. The conditional sprouting mechanism is implemented as follows. Each branch excepting root computes the average of its phenotypes and send it to its parental deme. These values are analyzed by the children_comparison $(x)$ procedure and compared with the phenotype of the best fitted individual $x$ distinguished from the parental deme. This procedure returns true if $x$ is sufficiently close to the existing child-demes. The branch reduction mechanism is omitted in Algorithm 1 for the sake of simplicity. All inter-deme messages are send and received asynchronously using buffers.

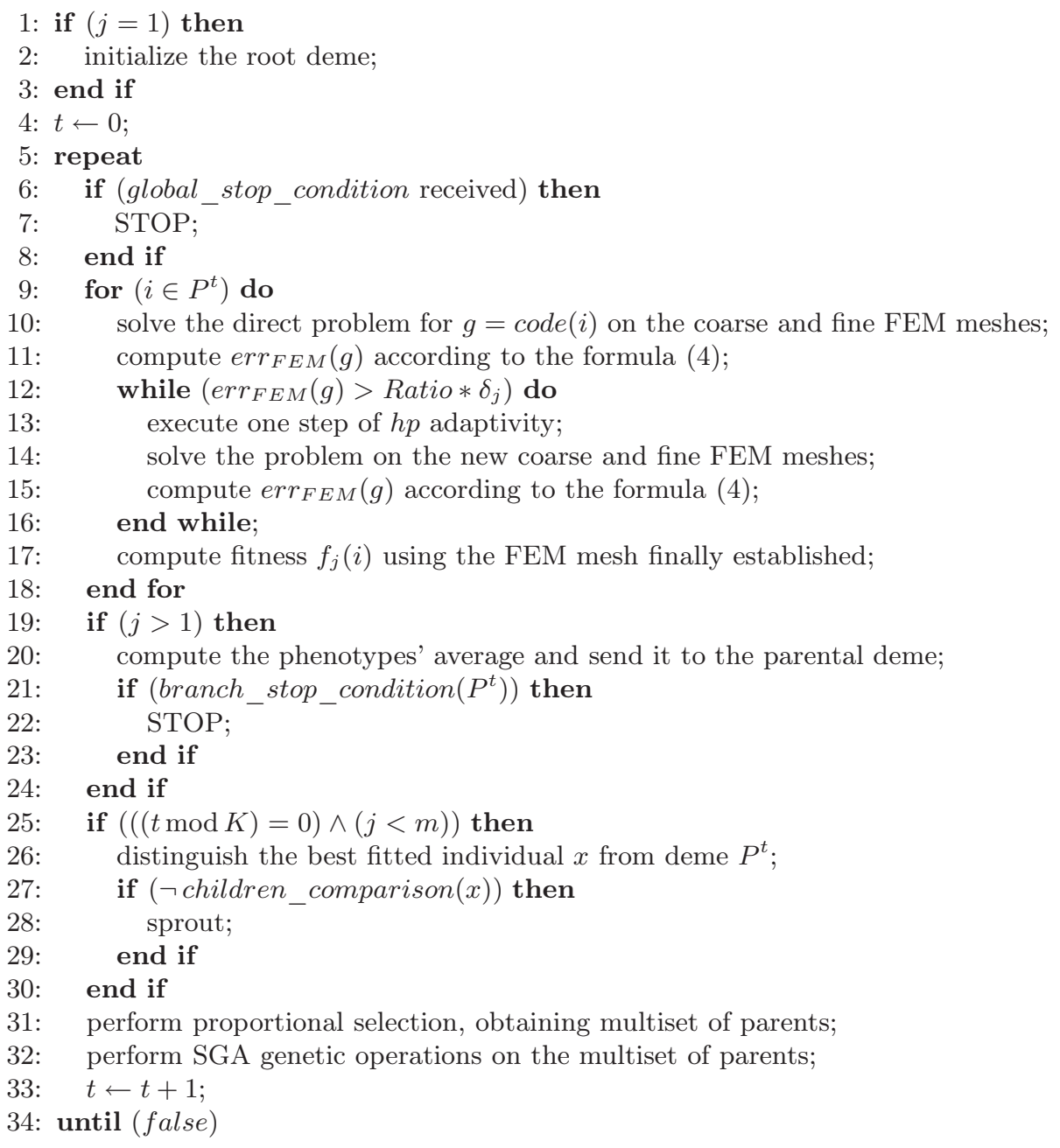

Algorithm 1: Pseudo-code of the $j$-th order deme $P$ in the $h p$-HGS tree 


\section{$4 \quad h p$-HGS Asymptotic}

The main goal of the asymptotic analysis presented below is to evaluate the efficiency of $h p$-HGS and compare its computational cost with the cost of two other strategies of solving inverse problem (5). The first strategy is the coupling of HGS with the same SGA engines in each branch as in $h p$-HGS, but with the fitness function $f_{m}$ computed as in $h p$-HGS leafs (e.g. by solving the direct problem with the maximum accuracy) and then induced to all branches of lower order. Notice, that such induction is well defined because of the nested HGS encoding (all phenotypes in branches of the order $j$ are also phenotypes in branches of the $j+1$ order). The second strategy is the single population SGA with the same fitness $f_{m}$ as previously. The size of the SGA population ensures the same initial local coverage of the admissible domain $\mathcal{D}$ by the SGA individuals as by individuals of each $h p$-HGS leaf.

We will intensively use the theory of the SGA heuristic (genetic operator) and its fixed points developed by Vose [15] as well as the convergence results of SGA sampling measures (see Schaefer [11, Chapter 4). Let $G_{j}: \Lambda^{r_{j}-1} \rightarrow \Lambda^{r_{j}-1}$ be the genetic operator (heuristic) of all branches (SGA demes) of the order $j$. It depends only on the number of genotypes $r_{j}$, fitness function $f_{j}$ and the genetic operations applied in branches of the order $j$. Moreover the unit simplex $\Lambda^{r_{j}-1} \subset \mathbb{R}^{r_{j}}$ stands for the set of frequency vectors of all possible demes of the order $j$. We assume that each genetic operator $G_{j}$ has the unique fixed point $z_{j}$ in $\Lambda^{r_{j}-1}$ that represent the limit population (i.e. the infinite cardinality population after the infinite number of genetic epochs). Moreover $z_{j}$ stands for the global attractor of $G_{j}$ on $\Lambda^{r_{j}-1}$ (i.e. $\forall x \in \Lambda^{r_{j}-1} \lim _{t \rightarrow+\infty}\left(G_{j}\right)^{t}(x)=z_{j}$ ).

Each deme $x \in \Lambda^{r_{j}-1}$ of the order $j$ may induce the probabilistic measure on $\mathcal{D}$ given by the density $\rho_{x} \in L^{p}(\mathcal{D}), p \geq 1$, so we may establish the mapping $\Psi_{j}: \Lambda^{r_{j}-1} \rightarrow \mathcal{M}(\mathcal{D})$ such that $\Psi_{j}(x)(A)=\int_{A} \rho_{x}(\xi) d \xi$ for each measurable set $A \subset \mathcal{D}$. The space $\mathcal{M}(\mathcal{D})$ collects all probabilistic measures over the admissible set $\mathcal{D}$. The densities $\rho_{x}, x \in \Lambda^{r_{j}-1}$ are piecewise constant on some subsets surrounding the phenotypes induced by the encoding of $j$-th order (e.g. on the Voronoi neighborhoods of phenotypes). Details of this construction may be found in Schaefer [11. Let us denote $\rho_{j}=\rho_{z_{j}}$ and $\psi_{j}=\Psi_{j}\left(z_{j}\right)$ for the sake of simplicity.

We assume that SGA governing the evolution of the $h p$-HGS branches of $j$-th order $j \in\{1, \ldots, m\}$ are well tunned (see Schaefer [11], Definition 4.63) i.e. the densities $\rho_{j}$ dominates on some closed sets $C^{j} \subset \mathcal{D}$ with the strictly positive Lesbegue measure (not necessary connected). Each set $C^{j}$ surrounds the local extremes to the objective of the inverse problem (5) and is contained in the basins of attraction of these extremes. Moreover we assume that $C^{1} \supset C^{2} \supset$ $, \ldots, \supset C^{m}$.

The analogous assumptions are made for the strategy in which the fitness $f_{m}$ is implemented in all HGS branches. The resulting quantities will be denoted as $\tilde{\rho}_{j}, \tilde{\psi}_{j}, \tilde{C}^{j}, j=1, \ldots, m$ in this case. 
Theorem 1. Given the above assumptions, the hp-HGS deme of the order $j_{0}=$ $2, \ldots, m$ survives with the probability not greater than $\kappa^{j_{0}}$ given by the formula:

$$
\kappa^{j_{0}}=\psi_{1}\left(C^{1}\right) \prod_{j=2}^{j_{0}} \frac{\psi_{j}\left(C^{j}\right)}{\psi_{j}\left(C^{j-1}\right)} .
$$

Sketch of the proof: Similarly to the proof of Hypothesis 1 in [10, it may be easily checked, that if the $h p$-HGS branch is sprouted from $C^{j-1}$ to $C^{j}$, then the probability of its surviving may be approximated by

$$
\psi_{1}\left(C^{1}\right)\left(1-\eta_{1}\right) \prod_{j=2}^{j_{0}} \frac{\psi_{j}\left(C^{j}\right)}{\psi_{j}\left(C^{j-1}\right)}\left(1-\eta_{j}\right)
$$

where $1-\eta_{j}$ is the probability that the branch of the order $j$ survives if it is sprouted inside $C^{j}$. If the deme of the order $j$ is sprouted outside $C^{j}$, then the probability of its surviving is $\eta_{j}$. This value becomes arbitrary small after the sufficient number of genetic epochs which is the issue of well tuning of this branch, and the inclusion $C^{j} \subset C^{j-1}$.

Lemma 1. Under the conditions similar to those assumed in the Theorem 1 the probability of surviving the deme of the order $j_{0}=2, \ldots, m$ in the HGS tree is not greater then

$$
\tilde{\kappa}^{j_{0}}=\tilde{\psi}_{1}\left(\tilde{C}^{1}\right) \prod_{j=2}^{j_{0}} \frac{\tilde{\psi}_{j}\left(\tilde{C}^{j}\right)}{\tilde{\psi}_{j}\left(\tilde{C}^{j-1}\right)}
$$

for the strategy in which the fitness $f_{m}$ is implemented in all HGS branches.

Lemma 1 generalizes the Hypothesis 1 in [10] to the case of HGS branches of the arbitrary order $j_{0}=2, \ldots, m$. Its proof is analogous to the proof of the theorem 11 above.

Corollary 1. Let $a_{1}<a_{2}<, \ldots,<a_{m}$ are the averaged costs of solving direct problems for the individuals in $h p-H G S$ branches of the orders $1, \ldots, m$. The computational cost of the single genetic epoch in all hp-HGS branches may be approximated by

$$
\mu_{1} a_{1}+\sum_{j=2}^{m} \sharp \Omega_{s_{j-1}} \kappa^{j} \mu_{j} a_{j} .
$$

Proof: Using the theorem 1 we can evaluate the expected computational cost of the single genetic epoch of the $h p$-HGS branch of degree $j>1$ which equals: $\sharp \Omega_{s_{j-1}} \kappa^{j}$ - the expected number of demes of the order $j$ times the constant cardinality of each deme $\mu_{j}$ times the averaged cost $a_{j}$. The thesis is obtained by summing over all degrees of the $h p$-HGS tree.

The above results allow to formulate three practical corollaries which try to evaluate the efficiency of the new introduced strategy. 
Corollary 2. Let hp-HGS satisfies all assumptions of the theorem 1 and $S G A$ is applied to solving the same inverse problem with the fitness $f_{m}$. The expected number of individuals in hp-HGS leafs is smaller than the expected number of individuals in $S G A$, so the computational cost of the single genetic epoch in the $S G A$ search is greater then the analogous cost of processing hp-HGS leafs.

Proof: The expected number of individuals in the $h p$-HGS leafs is $\sharp \Omega_{s_{m-1}} \kappa^{m} \mu_{m}$. The local initial coverage of $\mathcal{D}$ by the SGA individuals will be the same as the initial coverage by the $h p$-HGS leaf individuals if the size of the SGA population equals $\sharp \Omega_{s_{m-1}} \mu_{m}$. Because all $h p$-HGS branches are well tunned then $\kappa^{m}<1$.

Corollary 3. If the $h p-H G S$ and HGS branches are similarly well tuned i.e. $\tilde{\psi}_{j}\left(\tilde{C}^{j}\right) \cong \psi_{j}\left(C^{j}\right), j=1, \ldots, m$, then the cost of the single genetic epochs in hp-HGS branches is smaller then the analogous cost in HGS branches.

Proof: We can easily observed, that the assumption of the corollary implies $\tilde{\kappa}^{j} \cong \kappa^{j}$. The cost of the single genetic epoch of the HGS root is $\mu_{1} a_{m}$ while in the $h p$-HGS root equals $\mu_{1} a_{1}$. The cost of the single genetic epoch of the HGS branch of the order $j>1$ equals $\sharp \Omega_{s_{j-1}} \mu_{j} \tilde{\kappa}^{j} a_{m}$ while in the $h p$-HGS branch is $\sharp \Omega_{s_{j-1}} \mu_{j} \kappa^{j} a_{j}$. The thesis holds because $a_{j}<a_{m}$ for $j=1, \ldots, m-1$.

For sample, more detail comparison of HGS and $h p$-HGS computational costs the progression of the averaged costs of solving direct problems $a_{j}$ by the growing branch order $j$ will be evaluated. Here we assume the particular regression of the inverse problem error $\delta_{j}=\alpha^{j-1} \delta$, where $\delta$ and $\alpha<1$ are some positive parameters. Moreover we assume that the $h p$-HGS error by solving direct problem decreases exponentially with respect to $n_{j}$ - the number of degrees of freedom that defines the final approximate space $V_{h, p}$. This assumption is motivated by theoretical considerations (see Babuška [1], 2]) and many test computations (see e.g. 4, 5], 14, 13]).

Corollary 4. Given the above assumptions the average cost $a_{j}$ of solving direct problem for individuals of the hp-HGS deme of $j$-th order is

$$
\mathcal{O}\left((\theta(j-1)+\beta)^{3 \gamma}\right)
$$

where the constants $\theta>0, \beta \geq 0$ and $\gamma>1$ depend on the inverse problem under consideration.

Proof: $>$ From the definition of $h p$-HGS algorithm (see Algorithm 1) err $_{F E M}=$ $\delta_{j}$ Ratio $=\alpha^{j-1} \delta$ Ratio if we solve the direct problem for the individual in the branch of the $j$-th order, $j=1, \ldots, m$. Assuming the exponential decrement of the $h p$-FEM error, $\operatorname{err}_{F E M}$ may be also expressed as $\operatorname{err}_{0} \exp \left(-C\left(n_{j}\right)^{\frac{1}{\gamma}}\right)$ where $\operatorname{err}_{0}>1$ is the maximum error larger or equal then one established for the fitness computation in the root deme and $C>0, \gamma>1$ are some constants. Comparing both expressions we have $(\theta(j-1)+\beta)^{\gamma}=n_{j}$ where $\theta=-\frac{\ln \alpha}{C}$ 
and $\beta=\frac{\ln e r r_{0}-\ln (\delta \text { Ratio })}{C}$. The first constat $\theta$ is stritcly positive, because $C>0$ and $\alpha<1$ while $\beta \geq 0$ follows from the condition of the $h p$-HGS strategy (see Algorithm 1, line 12). Finally, the well known dependency $a_{j}=\mathcal{O}\left(n_{j}^{3}\right)$ (see e.g. Demkowicz [4]) motivates the thesis.

\section{Conclusions}

- Solving the inverse parametric problems is usually a difficult and time consuming numerical task. The sophisticated global optimization strategies have to be applied in order to find multiple solutions, keeping the memory and computational costs at the acceptable level.

- The $h p$-HGS strategy presented in this paper offers two ways to decrease the computational and memory costs by solving inverse parameter problems. Firstly it is obtained by decreasing of the number of the objective calls by using the adaptation of the inverse problem accuracy (HGS strategy). Secondly, the cost of the direct problem solution which is necessary for computing the particular value of the objective function is decreased by the proper scaling of the FEM error using the $h p$ adaptation technique.

- The theoretical results of the $h p$-HGS analysis allow to evaluate the expected computational cost of this strategy (Corollary 1). Moreover it was shown that its computational cost is less then the cost of the single population SGA algorithm (Corollary 2) as well as the cost of the HGS strategy without the FEM error scaling (Corollary 3). The sample formula that allow the more detailed cost comparison was drown for the second case (Corollary 4).

- No matter how SGA with the binary encoding is sometimes criticized as a tool for solving optimization problems in the continuous domains, it is used in each branch of the first version of $h p$-HGS mainly because the theoretical results that characterize its asymptotic behavior are available. We plan to design the next version of $h p$-HGS which will be based on the hierarchic genetic strategy with the real number encoding (see [1], 16]).

Acknowledgements. The part of the work was performed within COST action P19 and supported by the MNiSzW, project nr. COST/203/2006.

\section{References}

1. Babuška I., Guo, B.: The $h p$-version of the finite element method, Part I: The basic approximation results. Comput. Mech. 1, 21-41 (1986)

2. Babuška, I., Guo, B.: The $h p$-version of the finite element method, Part II: General results and applications. Comput. Mech. 1, 203-220 (1986)

3. Ciarlet, P.: The Finite Element Method for Elliptic Problems. Society for Industrial \& Applied Mathematics, Philadelphia (2002) 
4. Demkowicz, L.: Computing with $h p$-Adaptive Finite Elements. One- and TwoDimensional Elliptic and Maxwell Problems, vol. I. Chapmann \& Hall / CRC Applied Mathematics and Nonlinear Science (2006)

5. Demkowicz, L., Kurtz, J., Pardo, P., Paszyński, M., Rachowicz, W., Zdunek, A.: Computing with hp-Adaptive Finite Elements. Frontiers: Three-Dimensional Elliptic and Maxwell Problems with Applications, vol. II. Chapmann \& Hall / CRC Applied Mathematics and Nonlinear Science (2007)

6. Kołodziej, J.: Hierarchical Strategies of the Genetic Global Optimization. PhD Thesis, Jagiellonian University, Faculty of Mathematics and Informatics, Kraków, Poland (2003)

7. Kołodziej, J., Jakubiec, W., Starczak, M., Schaefer, R.: Identification of the CMM Parametric Errors by Hierarchical Genetic Strategy Applied. In: Burczyński, T., Osyczka, A. (eds.) Solid Mechanics and its Applications, vol. 117, pp. 187-196. Kluwer, Dordrecht (2004)

8. Momot, J., Kosacki, K., Grochowski, M., Uhruski, P., Schaefer, R.: Multi-Agent System for Irregular Parallel Genetic Computations. In: Bubak, M., van Albada, G.D., Sloot, P.M.A., Dongarra, J. (eds.) ICCS 2004. LNCS, vol. 3038, pp. 623-630. Springer, Heidelberg (2004)

9. Schaefer, R., Barabasz, B., Paszyński, M.: Twin adaptive scheme for solving inverse problems. In: X Conference on Evolutionary Algorithms and Global Optimization, pp. 241-249. Warsaw Technical University Press (2007)

10. Schaefer, R., Kołodziej, J.: Genetic search reinforced by the population hierarchy. In: De Jong, K.A., Poli, R., Rowe, J.E. (eds.) Foundations of Genetic Algorithms 7, pp. 383-399. Morgan Kaufman Publisher, San Francisco (2003)

11. Schaefer, R. (with the chapter 6 written by Telega H.): Foundation of Genetic Global Optimization. Springer, Heidelberg (2007)

12. Paszyński, M., Barabasz, B., Schaefer, R.: Efficient adaptive strategy for solving inverse problems. In: Shi, Y., van Albada, G.D., Dongarra, J., Sloot, P.M.A. (eds.) ICCS 2007. LNCS, vol. 4487, pp. 342-349. Springer, Heidelberg (2007)

13. Paszyński, M., Demkowicz, L.: Parallel Fully Automatic hp-Adaptive 3D Finite Element Package. Engineering with Computers 22(3-4), 255-276 (2006)

14. Paszyński, M., Kurtz, J., Demkowicz, L.: Parallel Fully Automatic hp-Adaptive 2D Finite Element Package. Computer Methods in Applied Mechanics and Engineering 195, 711-741 (2006)

15. Vose, M.D.: The Simple Genetic Algorithm. MIT Press, Cambridge (1999)

16. Wierzba, B., Semczuk, A., Kołodziej, J., Schaefer, R.: Genetic Strategy with real number encoding. In: VI Conference on Evolutionary Algorithms and Global Optimization, pp. 231-237. Warsaw Technical University Press (2003) 